\title{
Ancient disease, but not a thing of the past: Pott's paraplegia in an HIV-positive health-care worker
}

\author{
Leonardo F. Jurado ${ }^{1,2}$ \\ 1 Centro de Investigación UNINAVARRA-CINA, Fundación Universitaria Navarra, Neiva, Colombia \\ 2 Grupo de Investigación MICOBAC-UN, Departamento de Microbiología, Facultad de Medicina, Universidad \\ Nacional de Colombia, Bogotá, D.C., Colombia
}

\begin{abstract}
Each minute, three people die of tuberculosis in the world. This disease has affected the human race for about 70,000 years and now continues to be one of the biggest challenges in global health. In 2014, there were 9.6 million cases of active tuberculosis (12\% of them were HIV-positive) and 1.5 million of tuberculosis-related deaths $(0.4$ million among HIV-infected patients), which makes it the leading cause of death in people living with HIV (1).
\end{abstract}

We describe the case of a 40-year-old physician, who was working for a humanitarian organization in the Colombian jungle. He presented to the emergency room after developing a mass in the lumbar region $(6 \times 5 \mathrm{~cm})$ without local inflammatory changes, and subjective weight loss in the previous 20 days. $\mathrm{He}$ had a recent diagnosis of HIV infection and was already receiving highly active antiretroviral therapy (HAART). No fever or other systemic signs of an inflammatory response were found.

His chest $X$-ray was normal, his CD4 cell count was $346 \mathrm{cells} / \mu \mathrm{l}$ and his HIV viral load was undetectable. The tuberculin skin test showed a $20 \mathrm{~mm}$ cutaneous induration after 18 hours of application.

A contrast-enhanced spine MRI showed an illdefined lesion that affected predominantly the $L 4$ and L5 vertebral bodies and adjacent discs with epidural extension resulting in spinal canal stenosis. Additionally, there were multiple abscesses in the psoas muscles bilaterally, communicating posteriorly with a dorsal paraspinal and subcutaneous collection (figures 1 and 2).

A CT-guided needle aspiration of the abscesses was performed and the samples were sent for microbiological and cytological studies. Ziehl-

\section{Corresponding author:}

Leonardo F. Jurado, Departamento de Microbiología, Facultad de Medicina, Universidad Nacional de Colombia, Carrera $30 \mathrm{~N}^{\circ}$ 45-03, edificio 471, piso 3, Bogotá, D.C., Colombia

Teléfono: (312) 8737570

Ifjuradoz@unal.edu.co

Received: 08/08/16; accepted: 14/12/16

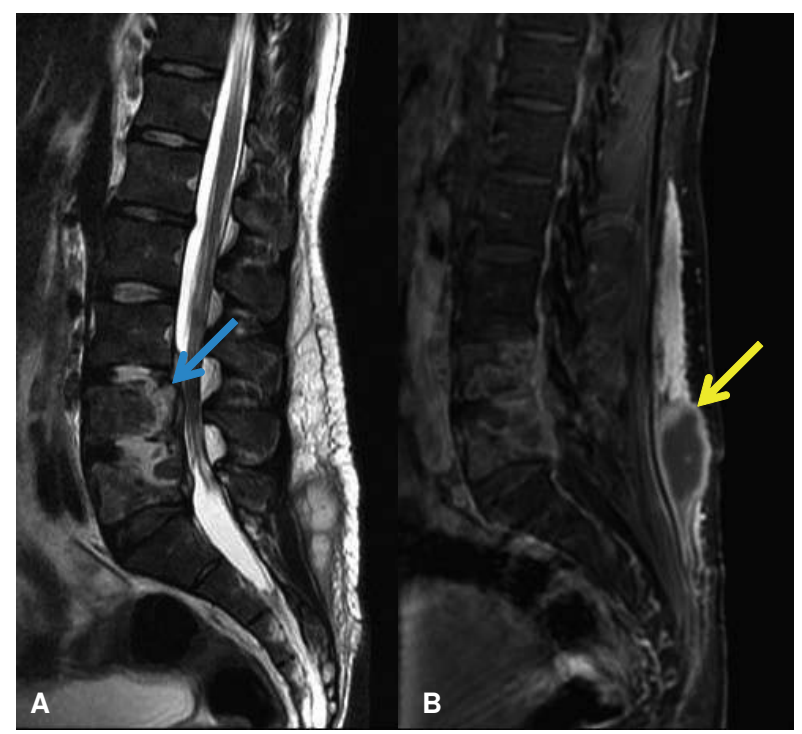

Figure 1. Sagittal T2-weighted (A) and post-contrast fat suppressed T1-weighted (B) images demonstrate L4 and L5 spondylodiscitis with anterior epidural phlegmon/abscess formation behind the L3-L5 vertebral bodies (blue arrow). Additional abscesses are seen in the prevertebral space (L4-L5) and dorsal subcutaneous soft tissues (L5/S1) (yellow arrow).

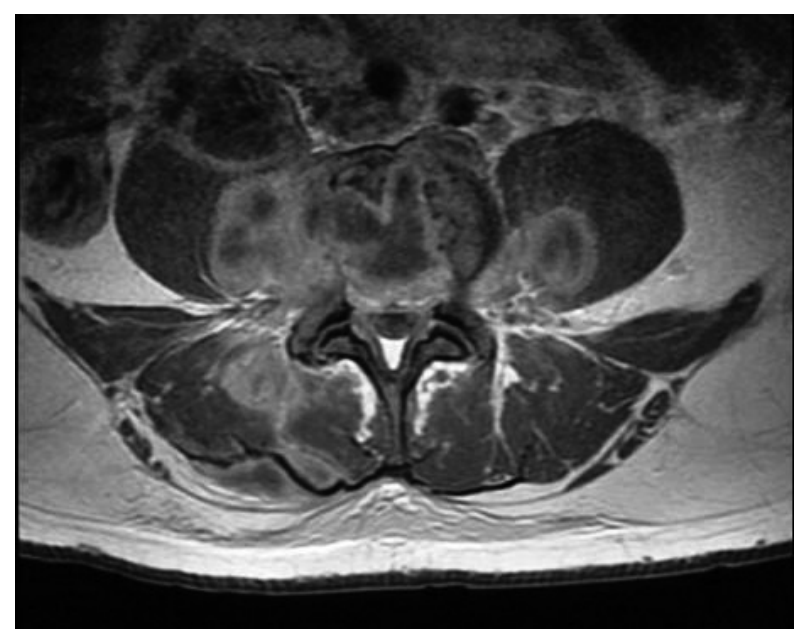

Figure 2. Axial non-fat suppressed T1-weighted image shows multiple abscesses affecting both psoas muscles and right posterior paravertebral muscles. 
Neelsen staining showed acid-fast bacilli, and a four drug regimen for TB treatment (rifampin, isoniazid, pyrazinamide and ethambutol) was started along with HAART. The solid media culture was positive after 20 days of incubation and, using biochemical and molecular tests, the isolate was identified as Mycobacterium tuberculosis susceptible to firstline drugs.

Due to the spinal mechanical compromise, surgical stabilization was performed. After three months of quad tuberculosis treatment, 10 months of dual (rifampin and isoniazid) therapy was prescribed, resulting in healing of the lesions and weight gain, without neurological sequelae to date.

Spinal tuberculosis was initially described in 1779 by Sir Percival Pott (2) as an uncommon, but highly destructive form of extrapulmonary tuberculosis. Around $10 \%$ of individuals who develop extrapulmonary tuberculosis have skeletal involvement, with the spine being the most frequently affected anatomical structure, followed by the hip and knee. Pott's disease accounts for approximately $50 \%$ of cases of skeletal tuberculosis (3).

Tuberculosis is a main cause of death in people infected with HIV, and its risk among people living with HIV is estimated to be 40 times greater than among those without HIV infection; conversely, individuals with a new diagnosis of tuberculosis are nearly 20 times more likely to be co-infected with HIV than those without tuberculosis (4).

Tuberculosis transmission between patients and health care workers inside and outside health care facilities has been reported in virtually every country in the world (5). Our patient, thus, had an increased risk of tuberculosis (infection and disease), because of his occupation.

Characteristically, the clinical features of spinal tuberculosis include local pain and tenderness, peripheral neurological symptoms, while constitutional symptoms (weight and appetite loss, night sweats, general discomfort) are present in $20-30 \%$ of the cases (6). However, our patient only presented with one of these symptoms (weight loss).

Destructive changes of the intervertebral disc and the adjacent vertebral bodies, with subsequent development of paravertebral abscess were seen in our patient. However, the typical collapse of the spinal elements and spinal kyphotic deformity (6) were not present (figures 1 and 2).
The evolution of spinal tuberculosis is slow and insidious, ranging from few months to a few years, the average duration varies from 4 to 11 months (6). This case is particularly interesting because of the rapid progression of the disease, as previously reported in HIV-positive patients (4). HIV infection is able to modify the initial clinical presentation and evolution of tuberculosis (4).

Tuberculosis remains a major cause of death worldwide and constitutes, along with malaria and HIV infections, a huge challenge for global health (7). The emergence and spread of tuberculosis/HIV coinfection, as well as the increasing prevalence of drug-resistant mycobacterial strains, further hinder the efforts for global tuberculosis control. Despite the increasing public awareness and clinical efforts aimed at reducing the global burden of tuberculosis, much remains to be done.

\section{Conflicts of interest}

The author declares no conflict of interest.

\section{Funding}

None.

\section{References}

1. World Health Organization. Global tuberculosis report 2014. Geneva: WHO; 2014.

2. Pott P. Remarks on that kind of palsy of the lower limbs which is frequently found to accompany a curvature of the spine, and is supposed to be caused by it, together with its method of cure, to which are added observations on the necessity and propriety of amputation in certain cases and under certain circumstances. London: J. Johnson; 1779.

3. Jurado LF, Murcia MI, Hidalgo P, Leguizamón JE, González LR. Phenotypic and genotypic diagnosis of bone and miliary tuberculosis in a HIV patient in Bogotá, Colombia. Biomédica. 2015;35:8-15. http://dx.doi.org/10. 7705/biomedica.v35i1.2275

4. Kwan C, Ernst J. HIV and tuberculosis: A deadly human syndemic. Clin Microbiol Rev. 2011;24:351-76. http://dx.doi. org/10.1128/CMR.00042-10

5. Jurado LF, Palacios DM, Álvarez J, Baldión M, Campos G. Pathological and molecular diagnosis in a case of primary laryngeal tuberculosis in a physician. Biomédica. 2014;34: 15-20. http://dx.doi.org/10.7705/biomedica.v34i1.1624

6. Moon MS. Tuberculosis of spine: Current views in diagnosis and management. Asian Spine J. 2014;8: 97-111. http://dx. doi.org/10.4184/asj.2014.8.1.97

7. Goldberg DE, Siliciano RF, Jacobs WR Jr. Outwitting evolution: Fighting drug-resistant TB, malaria, and HIV. Cell. 2012;148:1271-83. http://dx.doi.org/10.1016/j.cell.2012. 02.021 